\title{
Velocity Pick-up and Discharge Coefficient for Round Orifices with Cross Flow at
}

\section{Inlet}

\author{
Debin $\mathrm{Fu}^{1,2}$, John W. Chew ${ }^{2}$, Zixiang Sun ${ }^{2}$
}

\begin{abstract}
This study investigates the flow through round orifices with cross flow at the inlet. Emphasis is placed the change in tangential velocity component for orifices with low $L / D$. A definition of velocity pick-up is developed based on the orifice exit to cross flow tangential velocity ratio. Steady, incompressible and 3D computational fluid dynamics (CFD) models with the SST $k-\omega$ turbulent model are employed to calculate orifice flows with different geometrical and flow conditions. Stationary orifices and axial orifices in a rotating disk are considered. CFD solutions are compared with experimental results and published correlations for discharge coefficients, and good agreement is generally demonstrated. It is found that the non-dimensional velocity pick-up depends strongly on the ratio of characteristic times for flow to travel across the orifice in the tangential direction and that for the flow to pass through the orifice.
\end{abstract} A correlation of velocity pick-up as a function of this ratio is given.

\section{Keywords}

Discharge coefficient, Velocity pick-up, Tangential velocity, Orifice flow, CFD

\footnotetext{
${ }^{1}$ School of Aerospace Engineering, Beijing Institute of Technology, Beijing, People's Republic of China

${ }^{2}$ Thermo-Fluid Systems UTC, Faculty of Engineering and Physical Science, University of Surrey, Guildford, Surrey, GU2 7XH, UK

Corresponding author:

Debin FU, School of Aerospace Engineering, Beijing Institute of Technology, No.5, South Avenue, Zhongguancun, Haidian District, Beijing, China 100081

Email:fdb007@bit.edu.cn
} 


\section{Introduction}

The flow through orifices with cross flow at inlet is of interest in flow equipment such as turbines, compressors, centrifuges, pumps, motors, alternators, and generators. Typically, these orifices occur as stationary orifices in static structures, radial orifices in rotating shafts, and axial orifices in rotating discs. Since the equipment performance and component life are influenced by the orifice flow, the prediction of flow features in these systems and the calculation of the flow distribution are important to the system design process. In recent decades, the pressure loss in these systems and the mass flow of air through the orifice, characterized by the discharge coefficient, has been widely considered. Another feature of orifice flow is the change in the fluid's tangential velocity as it passes through the orifice. For example, as flow passes through an orifice in a rotating component its tangential or swirl velocity will change. The tangential velocity will affect vortex pressure losses and windage heating of the air in the system downstream of the orifice [1-3]. Thus the "velocity pick-up" of orifice flows is important, but there is little information available in the literature about how this might be estimated. For long orifices it is reasonable to assume that the fluid tangential velocity is close to that of the orifice, but this will not be the case for short orifices with low length to diameter ratios $(L / D)$.

For the estimation of discharge coefficient for orifice flow, geometric and flow parameters have been considered in detail, including the length to diameter ratio of the hole, hole inclination angle, hole entry or exit radiusing, pressure ratio, Reynolds number, rotation of the orifice, and presence of cross flow at inlet 
or outlet. For example, the discharge coefficient for flow through holes normal to a rotating shaft [4-5], compound-angle conical holes with cross-flow [6], axial orifices in a rotating disk [7], short orifices with radiused and chamfered inlet [8] are investigated recently. Hay et al [9] gave a review of discharge coefficient of turbine cooling holes and some methods correlating the discharge coefficients are reported by some researches [10-12]. In addition to discharge coefficients, some flow characteristics have been reported to explain orifice flow behaviour or describe specific phenomena. These involve the vena contracta, saddle-shaped velocity profile in near-field region for orifice flow, separation and reattachment flow [13-14]. Here we focus on the effect of inlet cross flow for sharp edged orifices aligned at $90^{\circ}$ to the inlet flow. Attention centres on low pressure ratio, high Reynolds number conditions at which the flow is not sensitive to these parameters.

A brief discussion of some previous studies of the effect of inlet cross-flow on orifice flow is relevant here. In 1969 Rohde et al. [15] published discharge coefficient measurements for orifices aligned at right angles to a fully developed mainstream duct flow. The proportion of the duct flow passing through the orifice could be controlled and results were presented for a range of orifice L/D ratios, sharp edged and rounded orifice inlets. Rhode et al. presented their data, together with that of Dittrich and Graves [16], as a discharge coefficient which assumed an inlet pressure equal to the total pressure in the duct plotted against velocity head ratio (VHR), defined as the difference between duct total pressure and orifice exit pressure divided by the dynamic pressure in the duct. Other workers have subsequently used experimental 
data from Rohde et al. and others to produce correlations of discharge coefficient for use in engineering calculations. For example, McGreehan and Schotsch [17] presented a correlation for use in turbomachinery internal flow network modeling taking into account inlet edge radius. The definition of discharge coefficient was based on assuming an orifice inlet pressure equal to the static pressure in the duct, and corresponds to that used in this paper. The effect of inlet cross flow was correlated through the ratio of inlet relative tangential velocity to the ideal orifice through flow parameter. In deriving their correlation McGreehan and Schotsch used data for rotating orifices from Meyfarth and Shine [18] and Grimm [19], in addition to Rohde et al.'s stationary orifice data.

More recently, computational fluid dynamics (CFD) has been established as a useful tool in estimating discharge coefficients. For example, Sousek et al. [20] show generally good agreement between CFD, using the SST $k-\omega$ model, for flow through an orifice in a rotating shaft. The maximum discrepancy in discharge coefficient between CFD and measurement is about $10 \%$ over a range of conditions. Also using the SST $k-\omega$ model, Da Soghe and Andreini [21] have studied discharge from a duct through multiple orifices aligned at right angles to the flow. CFD results from this study and earlier work by the same authors was used to produce a correlation for orifice discharge coefficient taking into account inlet cross flow, orifice pressure ratio, and orifice L/D ratio. Reasonable agreement of the correlation with measurements from Schulz et al. [22] was also demonstrated.

The present research extends previous work by presenting new experimental and CFD data, 
comparing discharge coefficients from various sources, and considering the tangential velocity "picked up" or changed by an orifice. Two typical cases, a stationary orifice with cross flow and flow through an orifice in a rotating disk, are considered. Moreover, an approximate correlation for velocity pick-up is put forward here. The model problems and detailed definition of velocity pick-up are given in section 2. CFD solutions are visualized and validated by comparison of discharge coefficients with experiment data and other workers results in section 3 and section 4. Results for velocity pick-up and some influences are discussed in section 5. Conclusions are given in section 6 .

\section{Definitions and models}

\subsection{Problems considered}

Two different orifice configurations, both having cross flow at the inlet, are considered. As shown in Figure 1(a), the first case is a stationary orifice in a duct side wall. In this case, the relative tangential velocity at orifice inlet $V_{\text {rel,i,t }}$ is approximately expressed as the duct flow average velocity. The second case is the flow through an axial orifice in a rotating disk as shown in Figure 1(b). Figure 1 indicates the relative and absolute orifice outlet velocities for each case. For simplicity, the subscript for absolute parameters is neglected below. A full list of notation is given in the appendix.

In these cases, orifices are assumed to be of round cross-sectional area with sharp edges, and ratios of orifice length to diameter in the range from 0.1 to 0.5 are considered. In this research, air at atmospheric conditions in the working fluid and the magnitude of flow Mach number considered is generally less than 
0.3. This condition corresponds to essentially incompressible flow.

\subsection{Definitions}

A definition of velocity pick-up is required that will be valid for both stationary and rotating orifices.

If the cross flow component of tangential velocity in a frame of reference moving with the orifice is zero at exit (i.e. $\left.V_{r e l, o, t}=0\right)$, the velocity pick-up $(V P)$ should be 1 . If the cross flow component of velocity at the exit equals that at the inlet $\left(V_{r e l, i, t}\right)$ then the value should be zero. Under these conditions, a suitable definition for the velocity pick-up is given as

$$
V P=1-\frac{V_{r e l, o, t}}{V_{r e l, o, t}^{*}}
$$

where $V_{r e l, o, t}^{*}$ is the exit tangential velocity that would occur if no tangential force was exerted on the flow as it passed through the orifice. For case (a) and (b) in Figure 1, this will equal the inlet tangential velocity $V_{r e l, i, t}$. Generally, the cross flow component or tangential velocity is related to the momentum conservation, so the following mass flow weighted velocity is utilized to define the average velocity at orifice inlet and outlet.

$$
V_{r e l, o, t}=\frac{\int_{A_{o}} \rho u_{r e l, o, t} u_{r e l, o, n} d A}{\int_{A_{o}} \rho u_{r e l, o, n} d A}
$$

and

$$
V_{r e l, i, t}=\frac{\int_{A_{i}} \rho u_{r e l, i, t} u_{r e l, i, n} d A}{\int_{A_{i}} \rho u_{r e l, i, n} d A}
$$

where $u$ is local velocity with $t$ and $n$ denoting tangential and normal components. 
The discharge coefficient is defined as the ratio of the actual flow divided by the flow for the ideal case,

i.e.

$$
C_{d}=\frac{\dot{m}_{\text {actual }}}{\dot{m}_{\text {ideal }}}
$$

The ideal flow mass rate $\dot{m}_{\text {ideal }}$ is calculated from the pressure difference across the orifice using the incompressible flow relations.

$$
\dot{m}_{\text {ideal }}=\rho A_{\text {orijce }} \sqrt{\frac{2 \Delta p}{\rho}}
$$

\subsection{Model geometry and computational domain}

As stated above, CFD is used to calculate the flow through orifices in this research. Mesh generation and flow solutions were carried out using the commercial software Gambit and Fluent [23]. Figure 2 shows geometries of CFD models used in the present work. For the purpose of comparison and validation of these models, most parameters correspond to experiment models.

For the flow through a stationary orifice, a straight square section duct model with an orifice on the top surface is utilized as shown in Figure 2(a). The geometry in this model corresponds to that studied experimentally by Glover [24]. The computational domain includes the duct, the orifice and surrounding region. To reduce the computing effort, symmetry boundaries are applied on the $z=0$ boundary which is shown in the figure. No-slip, no penetration boundary conditions have been applied on the duct and orifice surfaces. The flow inlet lies on the entrance of main duct, and mass flow with uniform velocity is 
specified here. Uniform pressure boundary conditions are applied to the duct outlet and outer boundaries of the surrounding region. The outlet to the surrounding region is at atmospheric pressure. The outlet pressure of the main duct is adjusted to control the pressure difference across the orifice.

Rotating disk models in the rotating reference frame are utilized here, representing the experimental configuration of Idris et al [7]. A schematic of the geometry and computational domain for this model is shown in Figure 2(b). The disk contains six holes at the same radius and equally spaced circumferentially. The inlet region consists of a cylindrical housing. This is followed by the orifice and an outlet cylindrical housing. Considering the repeating flow conditions, only a $60^{\circ}$ sector of the disc model is simulated. Periodic boundary conditions are imposed on the sector sides. As above, a mass flow boundary condition is specified at the inlet of the model and a uniform pressure boundary is applied to the outlet with atmospheric pressure. The inlet flow has a zero swirl component of velocity in the absolute frame of reference. No-slip and no penetration boundaries are applied on solid surfaces. The orifice model is given a spin equal to the rotational speed of the disk.

\subsection{CFD mesh and solution}

Block structured meshes are used in the present work. Denser grids are used in the vicinity of the orifice due to the high velocity gradients developed there. Considering both the model accuracy and speed, a limitation of the maximum value of mean dimensionless near-wall distance, i.e. $y_{\max }^{+}<5$, is satisfied for all cases. 
The turbulent model used is the SST $k-\omega$ model based on the eddy viscosity assumption. This effectively blends the formulation of the $k-\omega$ model in the near-wall region with the free-stream $k-\varepsilon$ model in the far field. As noted earlier this model has been used by other researchers for orifice flows. As mentioned above, incompressible flow solutions are obtained in this study. The fluid properties assumed correspond to air at standard atmospheric conditions.

A second-order accurate segregated solver using the SIMPLE pressure correction algorithm is used to obtain the CFD solutions. Under-relaxation is employed to ensure convergence. All of the results presented here are believed to have properly converged to a steady state. All residuals scaled by initial values reach $10^{-3}$ is a necessary condition for convergence. Most solutions showed considerably greater reduction in residuals than this. In addition, it has been confirmed that relative errors in the force/momentum balances for orifice flows in all directions are acceptably small within 5\%.

\section{Discharge coefficients}

Measured and calculated flow data are compared in this section, providing validation of the CFD model and comparison of results from various sources.

\subsection{Stationary orifice models}

Glover's [24] test rig is shown in Figure 3. The working section accommodated interchangeable orifice parts to allow the investigation of variation in orifice length $(L)$ and diameter $(D)$. To obtain the mass flow rate through the orifice, the input flow rate in the experiment was controlled by a Bronkhorst 
High-Tech Flow Controller, and an orifice meter was used to measure downstream mass flow rate. Static pressures within the duct near orifices were determined at locations slightly upstream. A throttle valve and suction pump were used to control duct exit pressure and prevent a high pressure blow-out.

The flow settling length in the $57 \mathrm{~mm}$ square section duct after the inlet is equal to approximately $1.7 \mathrm{~m}$. This allows ample length for flow development and is greater than the length assumed in the CFD model. Orifices with diameter of $15 \mathrm{~mm}$ and $25 \mathrm{~mm}$ were tested. In most cases, the pressure difference $\Delta p$ across the orifice is set to be in the range of $20 \mathrm{~Pa}$ to $60 \mathrm{~Pa}$ considering the accuracy of the equipment available. The standard errors of orifice discharge coefficient in this experiment were estimated as $1.9 \%$. The parameters used in the Glover's experiment and present simulation for stationary orifices are listed in Table 1.

Table 1 Parameters of Glover's experiment and present simulation

\begin{tabular}{|l|l|l|l|}
\hline Parameter & Unit & experiment & simulation \\
\hline Orifice diameter $D$ & {$[\mathrm{~mm}]$} & 15 & 15 \\
\hline Orifice length $L$ & {$[\mathrm{~mm}]$} & $5 ; 7.5$ & $0.15-15$ \\
\hline$L / D$ ratio & {$[-]$} & $0.3 ; 0.5$ & $0.1-1$ \\
\hline Pressure difference $\Delta p$ & {$[\mathrm{~Pa}]$} & $20-60$ & $2-2000$ \\
\hline Cross flow velocity $V_{c}$ & {$[\mathrm{~m} / \mathrm{s}]$} & $2.74-4.46$ & $2-40$ \\
\hline
\end{tabular}

Figure 4 plots discharge coefficients against velocity head ratio $(V H R)$. This figure compares McGreehan et al.'s correlation, Da Soghe et al.'s correlation, Dittrich et al.'s measurements, Rohde et al.'s 
measurements, Glover's measurements and the present CFD results. All results show a similar trend. Increasing $V H R$ reduces the effect of cross flow and tends to increase the discharge coefficient to a limiting value.

Generally, agreement between the different sets of results is good with differences of less than $10 \%$. Note that Da Soghe et al.'s correlation is based on CFD results for conditions in which $C_{d}>0.5$. Hence some departure from other results for very low VHR might be expected.

\subsection{Rotating orifices}

Measurements by Idris et al. and McGreehan et al.'s correlation are compared with CFD in Figure 5. Although the main focus of the present paper is for $L / D \leq 0.5$, it is encouraging to see reasonable agreement of CFD with Idris et al's measurements for an orifice in a rotating disk with $L / D=1.4$. For this case CFD and experiment agree within $4 \%$ of $C_{d}$. Note that in Figure 5 the abscissa is the cross flow to orifice flow velocity ratio, rather than the VHR used in Figure 4. The CFD results for this case were for a fixed mass flow given a mean orifice flow velocity of $67.2 \mathrm{~m} / \mathrm{s}$.

\section{Flow visualization}

Figure 6 shows some typical velocity vector plots for the two cases considered. Results are shown on planes passing through the orifice centre and are aligned to give comparable views for the three cases. The results for the stationary orifice are considered first as these do not include the effects of rotation and cross flow at exit, and are therefore clear. At high crossflow conditions (low $V_{n} / V_{c}$ ) Figure 6(a) shows 
that the flow impinges on the downstream edge of the orifice and that this leads to strong deflection of the flow as it passes through the orifice. As expected, high crossflow leads to a large separation zone on the upstream side of the orifice. At higher values of $V_{n} / V_{c}$ there is less deviation of the flow through the orifice. This condition also produces significant separation on the downstream side. Especially, the suction effect of the orifice leads to small tangential velocity component at orifice inlet and sometimes corresponds to negative $V P$.

At low crossflow conditions (high $V_{n} / V_{\text {rel,t }}$ ) the rotating orifice results in Figure 6(b) are qualitatively similar to those for the stationary orifice. Results for $V_{n} / V_{\text {rel,t }}=2$ in Figure 7(a), 7(b), and for $V_{n} / V_{\text {rel,t }}=5.2$ in Figure 7(a) also show some quantitative agreement. At high crossflow conditions, which also correspond to relatively strong rotational effects, more complex flows occur. These include substantial recirculation at the orifice exit, which leads to some unexpectedly high estimates of the velocity pick-up, VP. For such conditions the flow at the orifice exit and the value of VP are likely to depend significantly on the flow conditions downstream of the orifice.

\section{Velocity pick-up}

\subsection{Stationary orifices}

Figure 7 summarizes the results for velocity pick-up for the stationary orifice. Figure 7 (a) shows the CFD results from a series of tests for $L / D=0.1$, with $V P$ plotted against the ratio of orifice through flow velocity to inlet cross flow velocity $V_{n} / V_{c}$. For this seal, $V P$ is well below unity for all conditions. 
Generally $V P$ decreases as $V_{n} / V_{c}$ increases with the collapse of data showing independence of Reynolds number for these conditions. The region of small negative values of $V P$ around $V_{n} / V_{c}=2$ is associated with the flow being separated at the orifice exit, as noted from the flow visualization.

Figure 7 (b) shows the variation of $V P$ with $V_{n} / V_{c}$ for different $L / D$ values. As expected, increasing $L / D$ at a given velocity ratio increases $V P$. For higher $L / D$ values, $V P$ approaches unity at low values of $V_{n} / V_{c}$. At $L / D=1$, the assumption that $V P=1$ gives errors in $V_{o, t}$ less than $10 \%$ of the maximum of $V_{o, t}$ and $V_{n}$ for all conditions. i.e. $V_{o, t} / \max \left(V_{o, t}, V_{n}\right)<0.1$. For $L / D=0.5$, assuming $V P=1$ would give errors of up to $30 \%$ of the maximum of $V_{o, t}$ and $V_{n}$.

Attempting to account for the effects of both $V_{n} / V_{c}$ and $L / D$, Figure 7(c) plots the velocity pick-up against the ratio of characteristic times $T_{D} / T_{L}, T_{D}$ and $T_{L}$ are characteristic times for flow to cross the orifice and to pass through the orifice. The ratio is given by

$$
T_{D} / T_{L}=\left(D / V_{c}\right) /\left(L / V_{n}\right)=\left(V_{n} / V_{c}\right) /(L / D)
$$

Some collapse of data is shown in this figure for the cases of $L / D \leq 0.5$. With increasing $T_{D} / T_{L}$, $V P$ decreases and tends to a small value. For the region with small $T_{D} / T_{L}$, some significant differences exist between the results for different $L / D$.

\subsection{Rotating orifices}

A series of rotating disk orifice solutions with rotational speeds from 1000 RPM to 18000 RPM are obtained for different orifice length to diameter ratios. Figure 8 shows the results for $V P$ plotted against 
$V_{n} / V_{t}$ and $T_{D} / T_{L}$. As for the stationary orifice, for given $L / D, \quad V P$ decreases with increasing $V_{n} / V_{t}$

or $T_{D} / T_{L}$ and tends to a small value. Note that some values exceed 1 for small $T_{D} / T_{L}$ and $0.3 \leq L / D \leq 0.5$. These results are influenced by complex flows in the rotating orifice as discussed in section 4 .

\subsection{Correlation for velocity pick-up ratio}

As described above, velocity pick-up will be low for orifice flows with large $T_{D} / T_{L}$ and is of interest for orifices with low $L / D$. It has been shown that VP depends to a significant extent on flow conditions. Nevertheless a correlation of the present results is presented, as this may be useful in approximate engineering calculations.

A simplified analysis of the orifice, assuming a tangential force due to the stagnation of the tangential flow on the orifice inner surface, has been used to suggest a form for correlation of VP. Taking the tangential force to be proportional to $\rho V_{t}^{2} / 2$ times the elemental surface area, and assuming a constant through flow velocity component, the following expression for VP may be derived.

$$
V P=k_{1} /\left(k_{1}+T_{D} / T_{L}\right)+k_{2}
$$

where $k_{1}$ and $k_{2}$ are constants to be determined.

Figure 9 gives plots of $V P$ against $T_{D} / T_{L}$ for all numerical results with $L / D \leq 0.5$. Correlations of the form given by equation (7) are also included. For stationary orifice models, $k_{1}=2.85$ and $k_{2}=-0.093$; For rotating disk models, $k_{1}=3.9$ and $k_{2}=-0.15$; Although some scatter is 
noticeable, it is clear that $V P$ is reasonably correlated.

\section{Conclusion}

The flow through round orifices with cross flow at inlet was investigated in this research. Steady, incompressible and 3D CFD models with the SST $k-\omega$ turbulent model were employed to simulate the flows through stationary orifices, axial orifices in a rotating disk, and radial orifices in a rotating shaft. It was shown that the discharge coefficients of these orifices are in general agreement with other workers' experimental results and correlations. McGreehan and Schotsch's [18] empirically based correlation showed reasonable agreement with all data. Da Soghe and Andreini's [22] correlation based on CFD for quite different conditions.

Changes of the fluids tangential velocity when it passes through the orifice have been investigated using CFD. A definition of velocity pick-up ratio $(V P)$ has been developed based on the orifice exit to inlet tangential (or cross flow) velocity ratio. For short orifices $V P$ may be considerably less than 1 , indicating that significant tangential momentum is advected through the orifice. In rotating machinery applications this may affect vortex strength, and pressure distribution in cavities downstream of the orifice.

Velocity pick-up was shown to be a function of $T_{D} / T_{L}$, the ratio of characteristic times for flow to cross and to pass through the orifice. A correlation of results for $V P$ as a function of $T_{D} / T_{L}$ was produced, and may be used for approximate prediction. However, results show significant scatter about 
this correlation associated with rotational and downstream flow effects, so some caution is advised. The correlation was based on results for orifices with $0.1 \leq L / D \leq 0.5$, as this range was considered to be of meet practical interest. For $L / D=1$, the assumption $V P=1$ would give errors in the exit tangential velocity less than $10 \%$ of the maximum of the inlet tangential velocity and the through flow velocity.

\section{Acknowledgements}

This work has been conducted at the Thermo-Fluids Systems, University Technology Centre at the University of Surrey. Financial support from the China Scholarship Council for Dr. D. Fu as a visiting scholar and support from the University of Surrey are gratefully acknowledged.

\section{References}

[1] Etemad, M. R., Pullen, K., Besant, C. B. and Baines, N. Evaluation of windage losses for high-speed disc machinery. Proc Instn Mech Engrs, Part A, 1992, 206 (3): 149-157.

[2] Swain, R. and Preater, R. Investigation of windage heating effects on rotating components using pulsed ESPI. Optics and Lasers in Engineering, 1997, 26: 377-394.

[3] Fiebig, M. Embedded vortices in internal flow: heat transfer and pressure loss enhancement. Int. J. Heat and Fluid flow. 1995, 16: 376-388.

[4] Alexiou, A., Hills, N. J., Long, C. A., Turner, A. B., Wong, L. S. and Millward, J. A. Discharge coefficients for flow through holes normal to a rotating shaft. Int. J. Heat and fluid Flow, 2000, 21: 
701-709.

[5] Sousek, J., Pfitzner, M. and Niehuis, R. Experimental study of discharge coefficients of radial orifices in high-speed rotating shafts. Proceedings of ASME Turbo Expo 2010. GT2010-22691: 1-10.

[6] Taslim, M. E. and Ugarte, S. Discharge coefficient measurements for flow through compound-angle conical holes with cross-flow. International Journal of Rotating Machinery, 2004, 10: 145-153.

[7] Idris, A., Pullen, K and Barnes, D. An investigation into the flow within inclined rotating orifices and the influence of incidence angle on the discharge coefficient. Proc. IMechE Part A: J. Power and Energy, 2004, 218: 55-69.

[8] Dittmann, M., Dullenkopf, K. and Wittig, S. Discharge coefficients of rotating short orifices with radiused and chamfered inlets. Journal of Engineering for Gas Turbines and Power, 2004, 126: 803-808.

[9] Hay, N. and Lampard, D. Discharge coefficient of turbine cooling holes: a review. Journal of Turbomachinery, 1998, 120: 314-319.

[10] Rowbury, D. A., Oldfield, M. L. G. and Lock, G. D. A method for correlating the influence of external crossflow on the discharge coefficients of film cooling holes. Journal of Turbomachinery, 2001, 123: $258-265$.

[11] Huning, M. Comparison of discharge coefficient measurements and correlations for several orifice designs with cross-flow and rotation around several axes. Proceedings of ASME Turbo Expo Berlin, 2008. GT2008-50976: 1-14. 
[12] Idris, A. and Pullen, K R. Correlations for the discharge coefficient of rotating orifices based on the incidence angle. Proc. IMechE Part A: J. Power and Energy, 2005, 219: 333-352.

[13] Deo, R. C., Mi, J. and Nathan, G. J. The influence of nozzle-exit geometric profile on statistical properties of a turbulent plane jet. Experimental Thermal and Fluid Science, 2007, 32: 545-559.

[14] Ramamurthi, K. and Nandakumar, K. Characteristics of flow through small sharp-edged cylindrical orifices. Flow Measurement and Instrumentation , 1999, 10: 133-143.

[15] Rohde, J. E., Richards, H. T. and Metger, G. W. Discharge coefficients for thick plate orifices with approach flow perpendicular and inclined to the orifice axis, NASA Technical Note, NASA TN D-5467. 1969.

[16] Dittrich, R. T. and Graves, C. C. Discharge coefficient for combustor liner air-entry holes. I Circular Holes with Parallel Flow. NACA TN 3663, 1956.

[17] McGreehan, W. F. and Schotsch, M. S. Flow characteristics of long orifices with rotation and corner radiusing. Journal of Turbomachinery, 1988, 110: 213-217.

[18] Meyfarth, P. F. and Shine, A. J. Experimental study of flow through moving orifices. Journal of Basic Engineering, Dec. 1965: 1082-1083.

[19] Grimm, R. E. Fluid flow characteristics through orifices in enclosed rotating disks. M. S. Thesis, AFIT, 1967.

[20] Sousek, J., Riedmuller, D. and Pfitzner, M. Experimental and numerical investigation of the flow 
field at radial holes in high-speed rotating shafts. Proceedings of ASME Turbo Expo 2012, GT2012-68209.

[21] Da Soghe, R. and Andreini, A. Numerical characterization of pressure drop across the manifold of turbine casing cooling system. Proceedings of ASME Turbo Expo 2012, GT2012-68787.

[22] Schulz, A., Gritsch, M., and Wittig, S. Effect of crossflows on the discharge coefficient of film cooling holes with varying angles of inclination. ASME, Turbo Expo, 2001-GT-0134.

[23] Ansys. Fluent. Fluent 6.3 Documentation.

[24] Glover, S. M. Discharge coefficient and flow measurement for orifices with crossflow. Final Year Project Report, University of Surrey, 2011.

\section{Appendices}

Notation

$A$ surface area

$C_{d}$ discharge coefficient

$D$ diameter of orifice

$F_{p} \quad$ stagnation force on orifice surface

$k_{1}$ parameter for velocity pick-up ratio

$k_{2}$ parameter for velocity pick-up ratio 


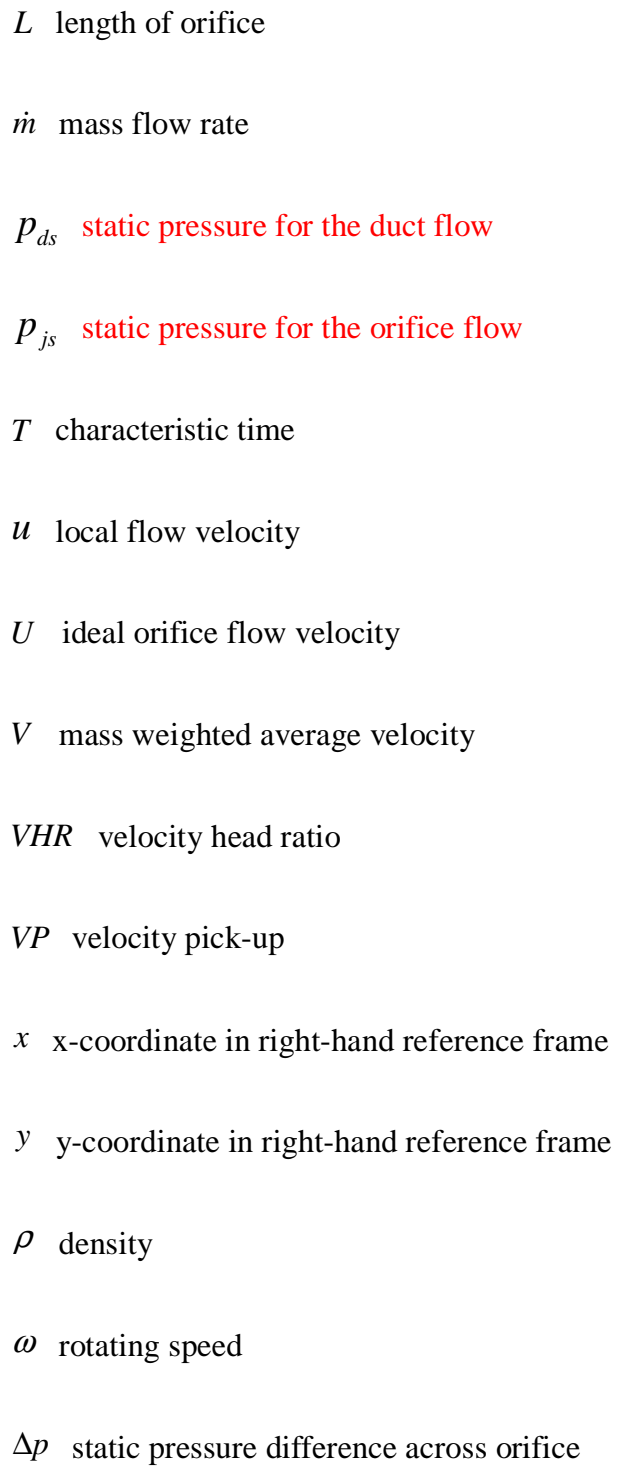

\section{Subscripts}

abs parameters in the absolute frame 
$c$ parameters in the cross flow direction

$D$ expresses the characteristic flow parameter across the orifice

$i$ parameters at the inlet of orifice

$L$ expresses the characteristic flow parameter pass through the orifice

$n$ parameters in the orifice axial direction

$o$ parameters at the outlet of orifice

rel parameters in a relative reference frame which fixed with the orifice

$t$ parameters in the tangential direction

$T$ parameters based on total pressure 

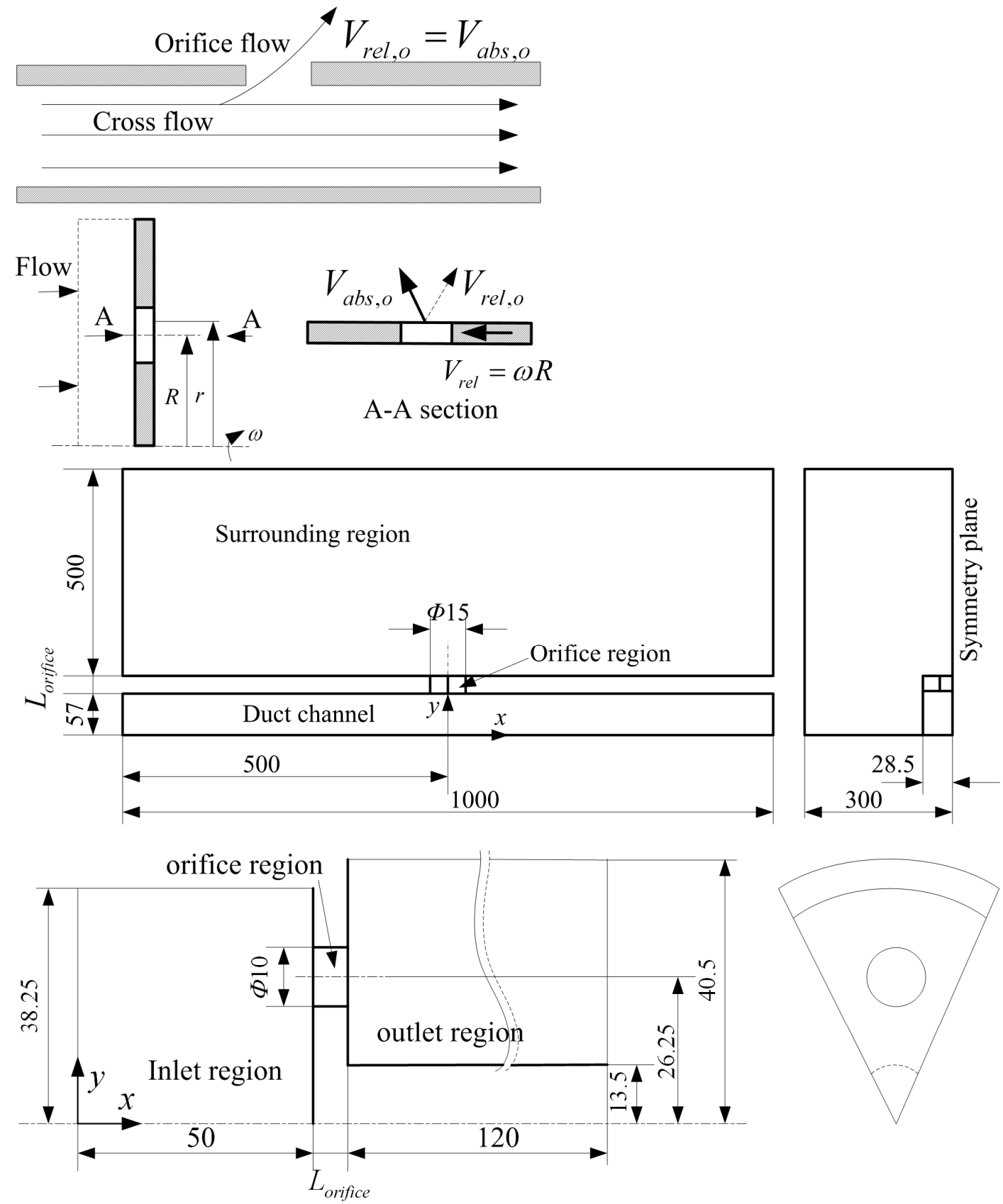

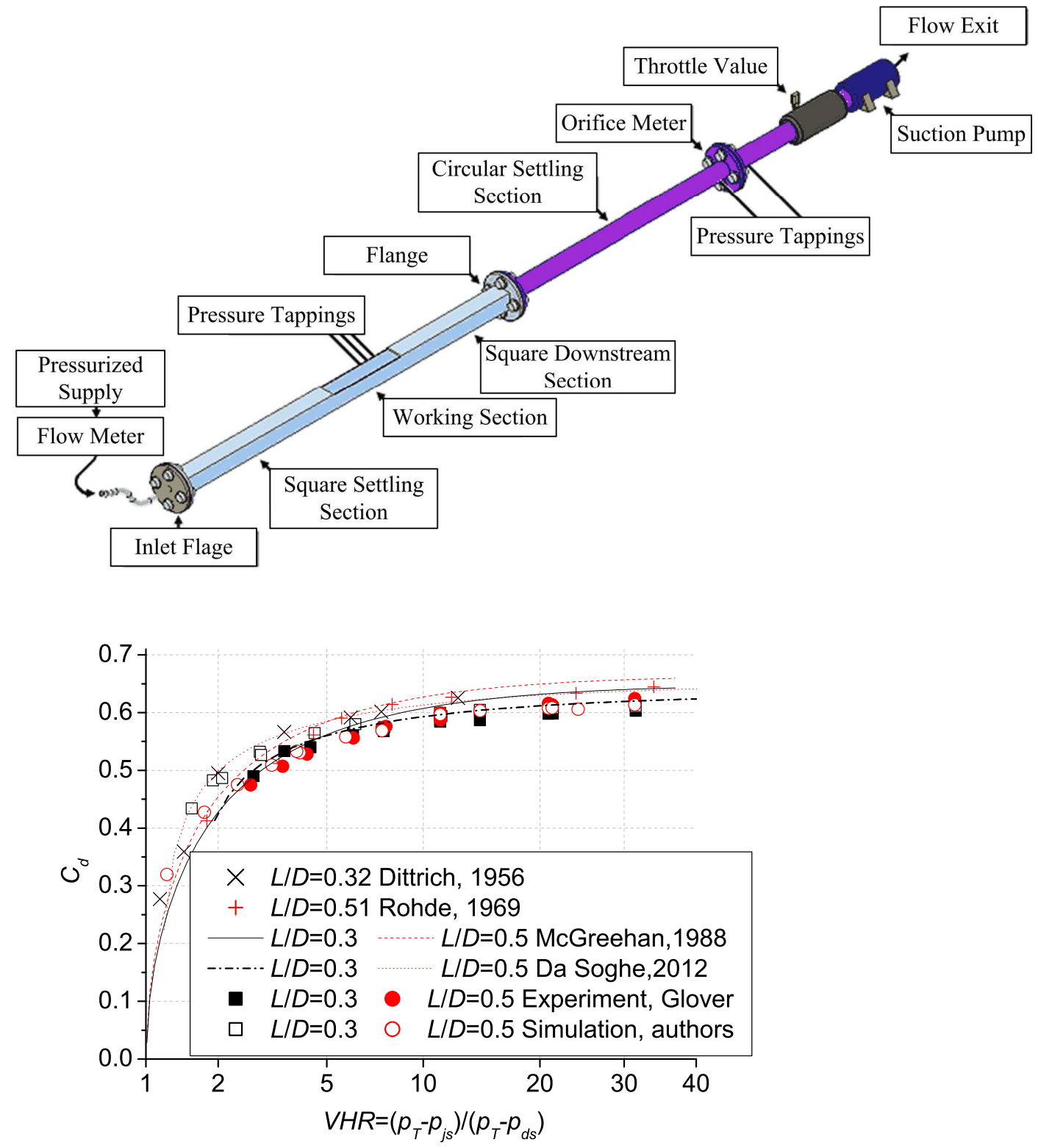

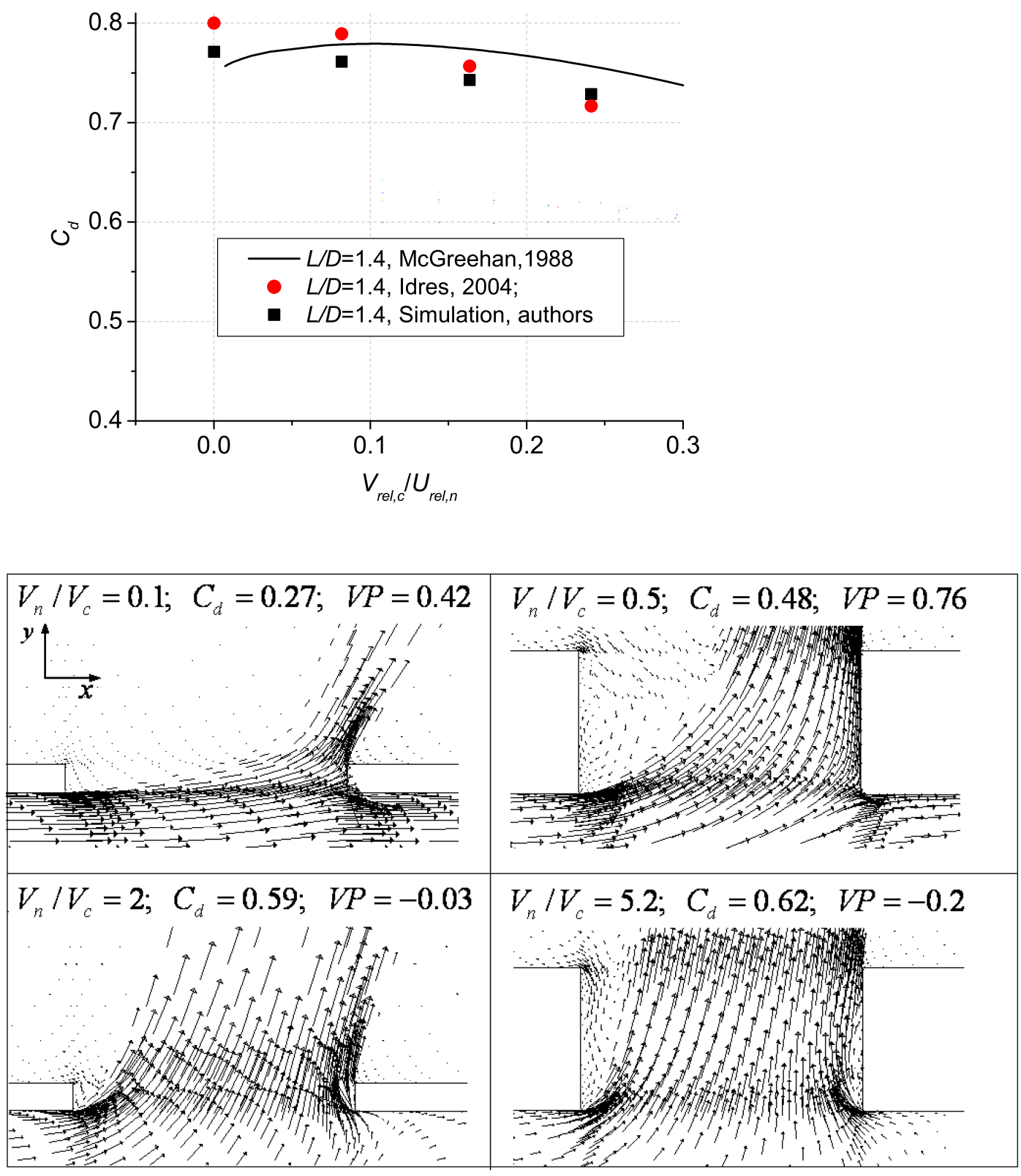

$L / D=0.1$

$L / D=0.5$ 


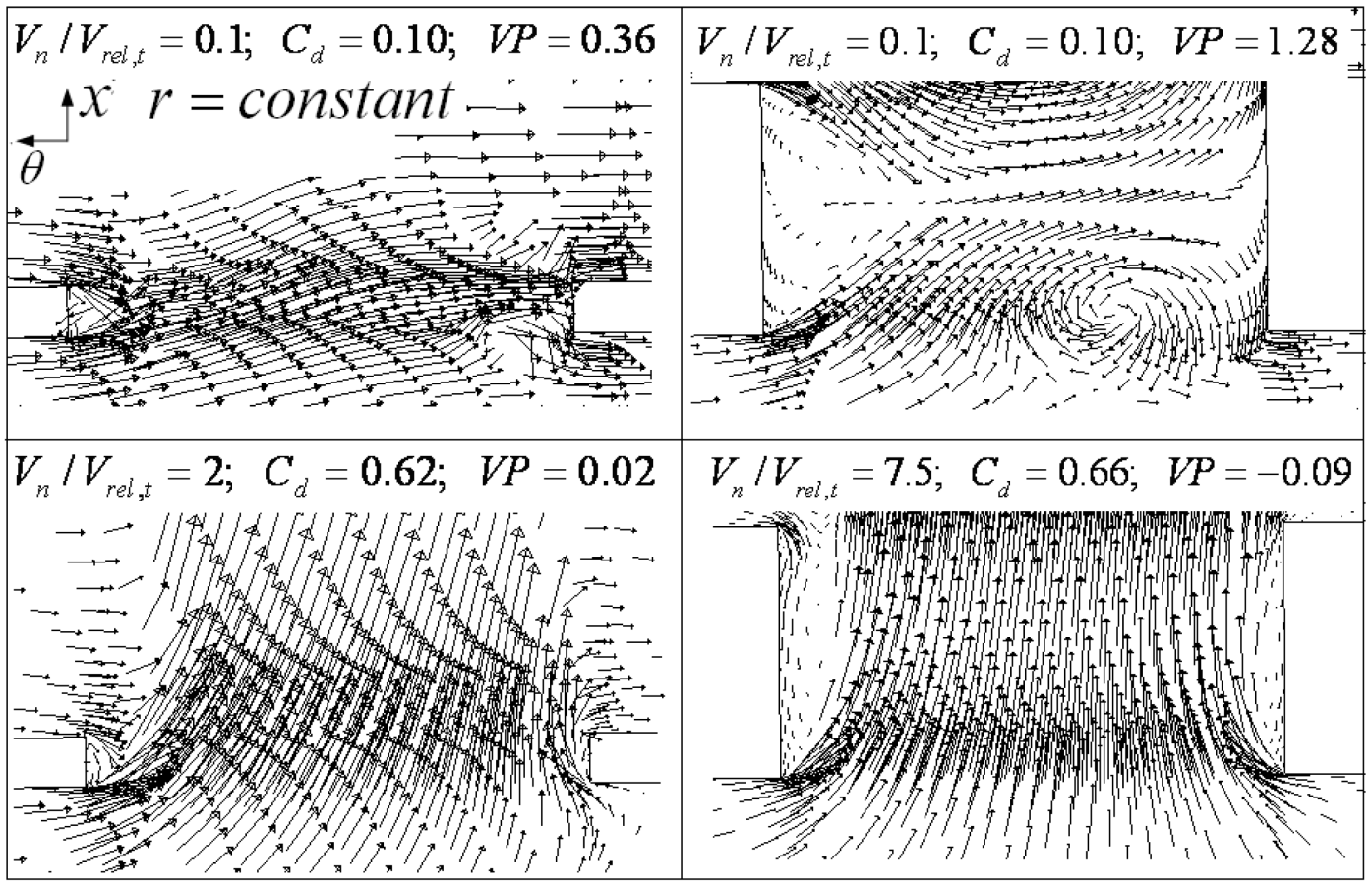

\section{$L / D=0.1$}

$L / D=0.5$

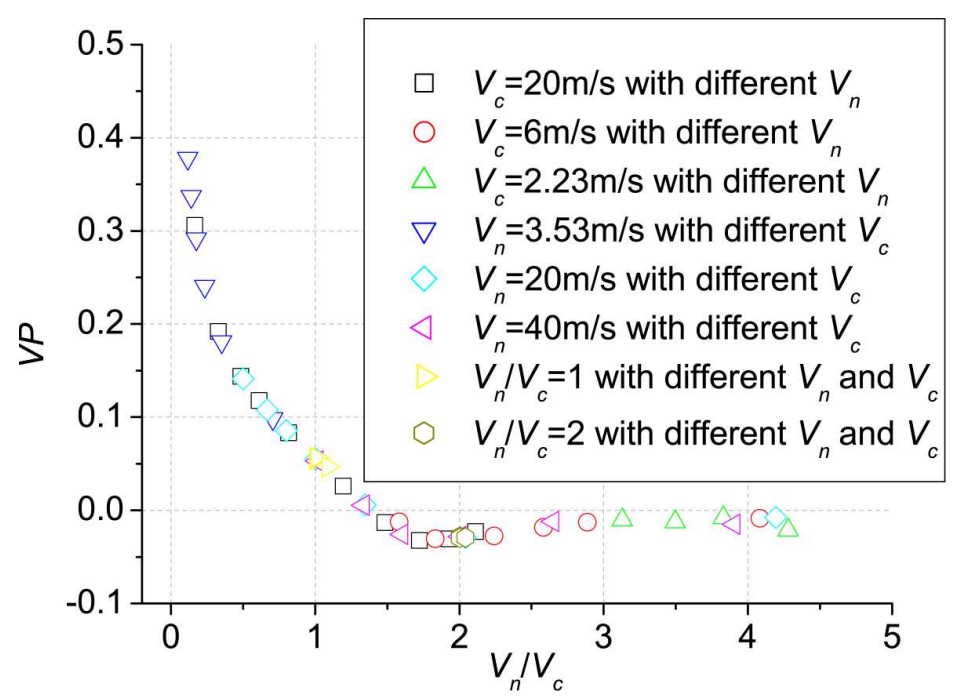




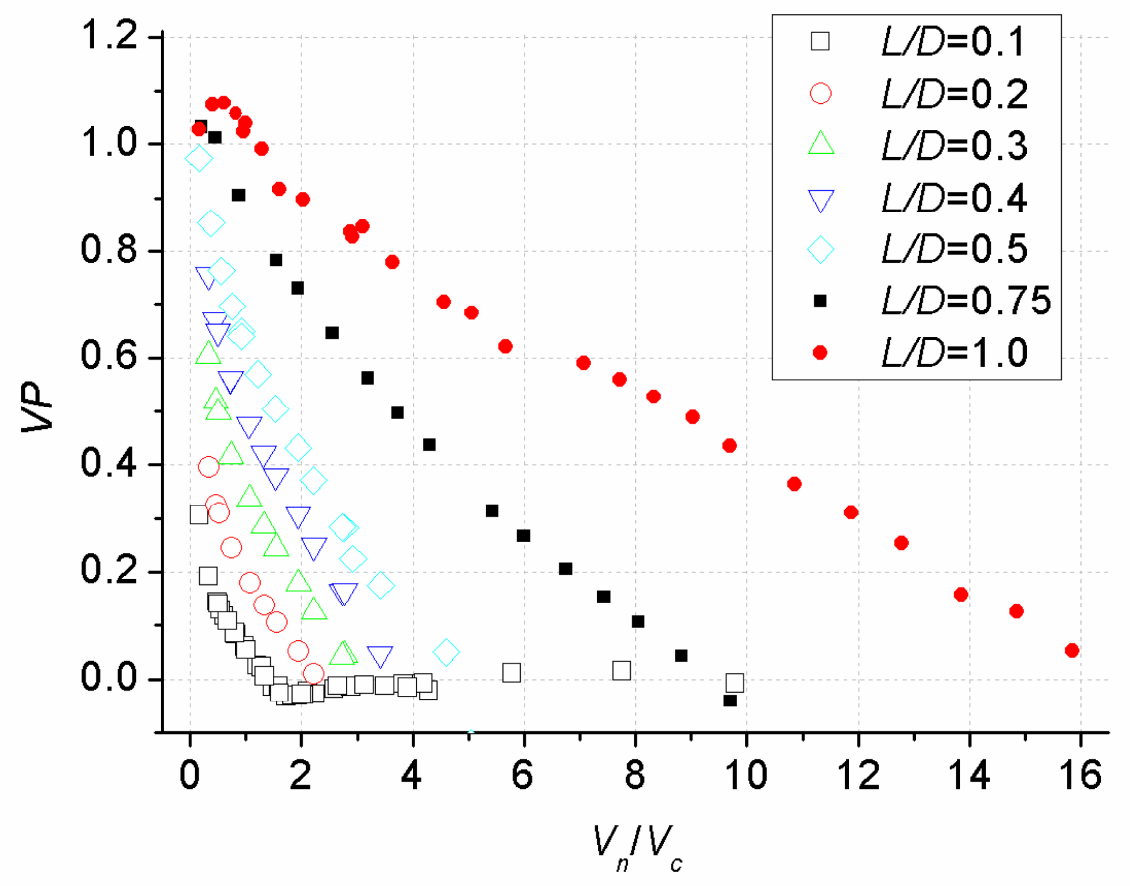




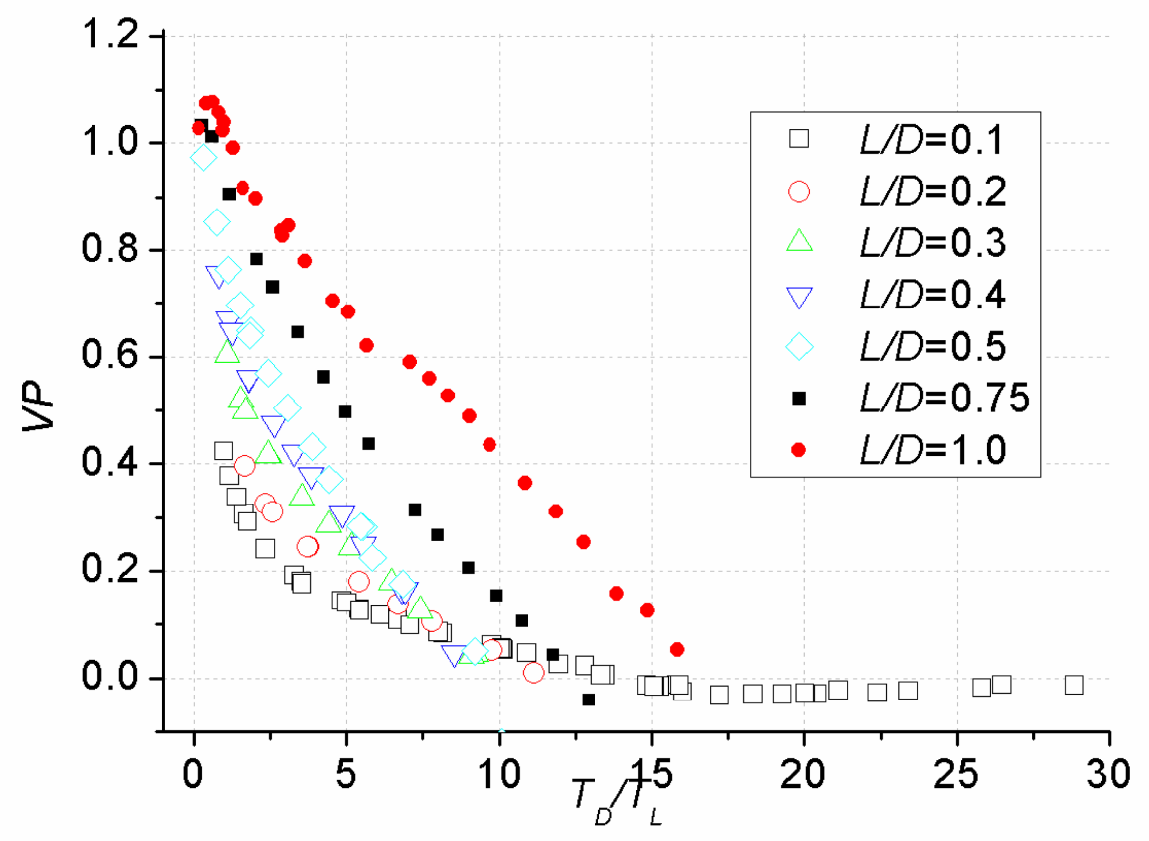




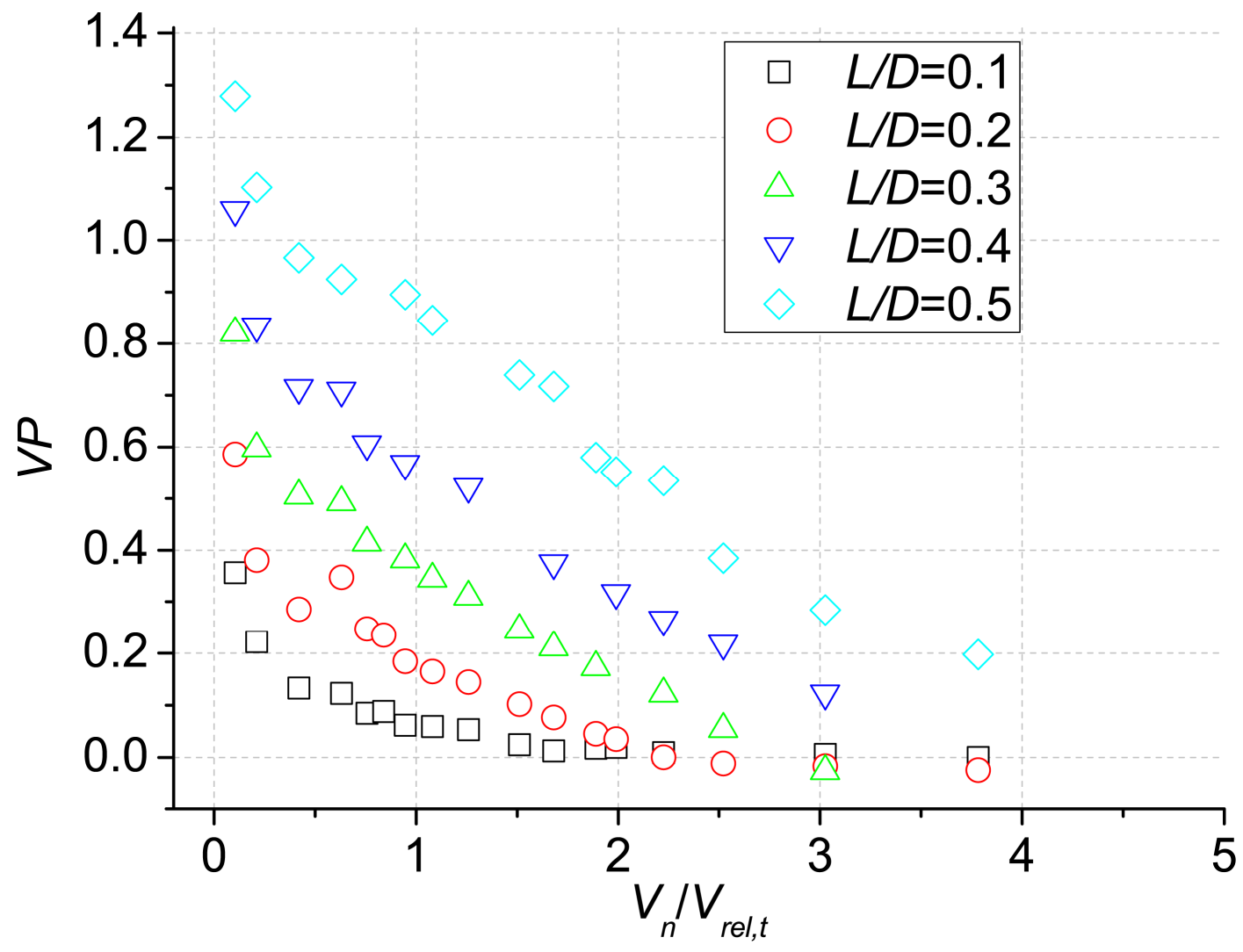




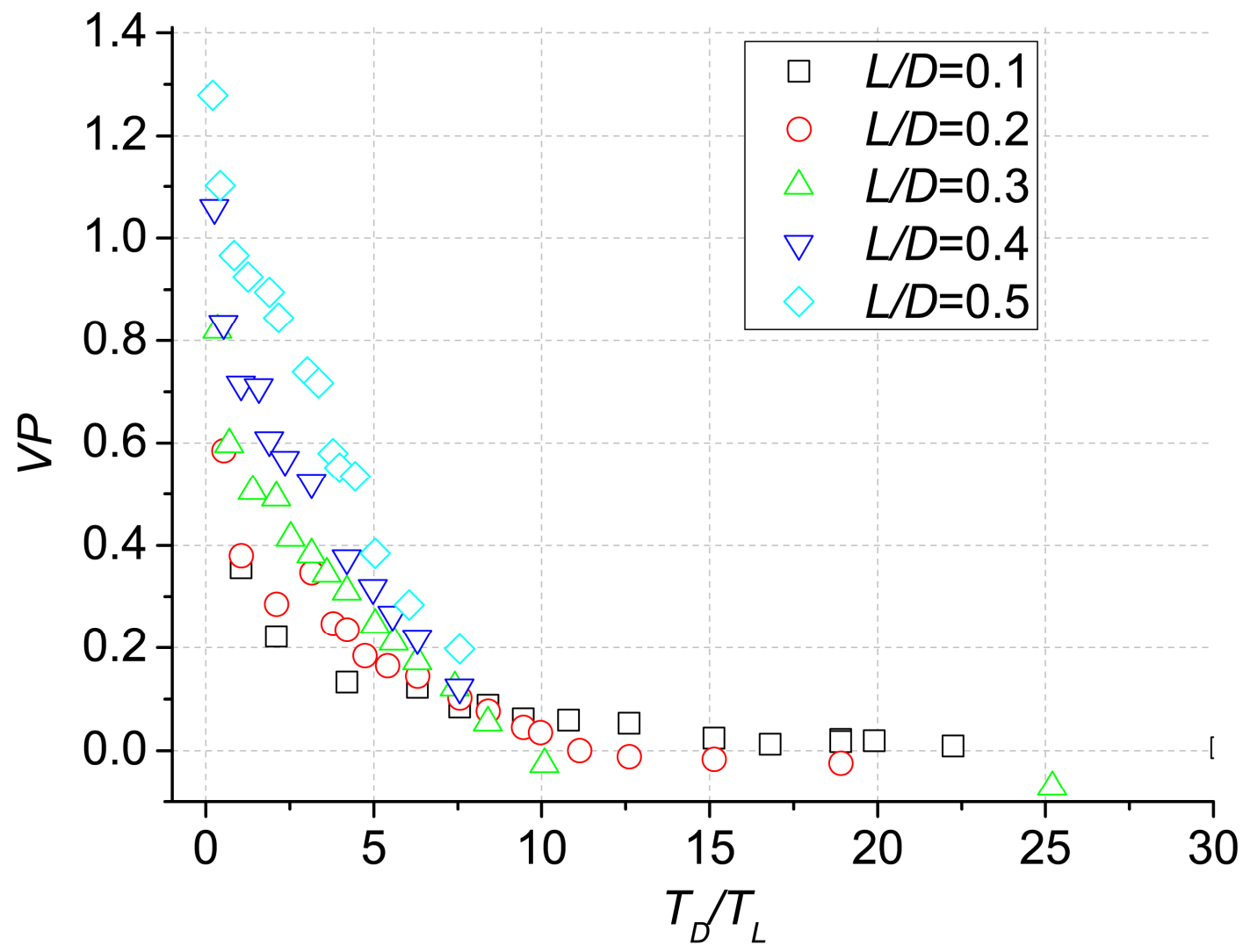




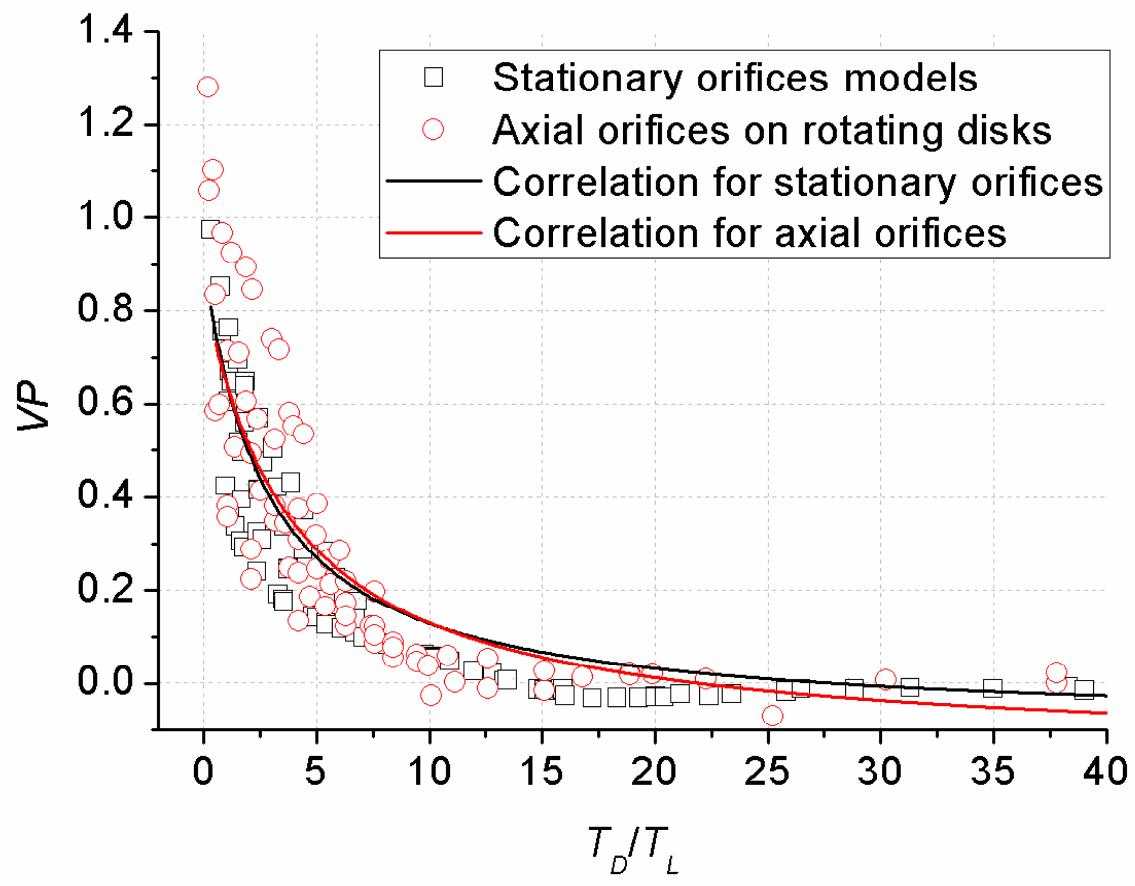

